\title{
Raison et sentiment
}

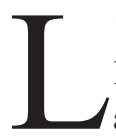

'affaire fit scandale en 1936 dans les milieux rationalistes de Grande-Bretagne. Sotheby avait entrepris cette année-là de disperser cent vingt manuscrits d'alchimistes ayant appartenu au plus grand des savants britanniques. Sir Isaac Newton en personne ! Keynes se porta acquéreur pour le compte de l'université de Cambridge. Il calma la polémique en décrivant le long chemin qui va de la magie à la science, de l'irrationnel au rationnel. « Newton ne fut pas le premier de l'âge de raison écritil. Il était le dernier des Babyloniens et des Sumériens, le dernier grand esprit à contempler le monde visible et intelligible avec les mêmes yeux que ceux qui commencèrent à construire notre héritage intellectuel il y a dix mille ans. "

Le dernier, vraiment? Les scientistes de 1936 ignoraient les chemins de traverse qui attendaient « l'âge de raison », hier avec le champignon d'Hiroshima, aujourd'hui avec les laboratoires du transhumanisme. Déjà au temps des Lumières, le mépris d'un Diderot pour les croyances pseudo-scientifiques des générations précédentes trahissait la croyance naïve en une prochaine « fin de l'Histoire » de l'obscurantisme. Il oubliait que la science ne vit pas enfermée dans une bulle coupée du monde. Qu'elle est au contraire immergée dans le monde des humains. Un monde de désir et de sentiment, sans frontière établie entre le rationnel et l'irrationnel.

Le sujet est d'actualité aujourd'hui avec la révolution numérique et l'effet de table rase qu'elle produit tout à la fois sur le travail, le commerce, l'entreprise et la cité. Par un étonnant retournement, on découvre que pour s'y préparer, mieux vaut s'affranchir des 
normes héritées des deux dernières Révolutions industrielles et revivre les basculements de périodes plus anciennes.

Un exemple encore proche: l'ère de la Réforme et de la Contre-Réforme. En 1600, l'Europe de l'Édit de Nantes voit arriver à maturité la génération des Galilée, Grotius, Monteverdi, Mersenne ou Caravage. Un homme va alors bâtir un véritable « réseau social» qui se déploie sur tout le continent européen avec des connexions en Asie, Afrique et MoyenOrient. Il prend la forme d'un immense réseau postal, sécurisé, indépendant des pouvoirs de l'Université, de l'Église et des États. Une plate-forme ouverte aux savants et aux juristes de Londres, Paris ou Amsterdam mais aussi aux poètes, aux grands voyageurs sans oublier les commerçants internationaux du port de Marseille. Car c'est d'Aix-en-Provence que le réseau est animé par une gloire mondiale à l'époque et un inconnu aujourd'hui :
Nicolas-Claude Fabri de Peiresc, conseiller au Parlement de Provence.

Ami de Galilée, Peiresc plaide sans succès sa cause à Rome mais parvient à contourner le plus grand portail d'information de la Renaissance finissante, la bibliothèque vaticane. Selon l'historien Peter N. Miller «à travers ses 70000 lettres et notes, Peiresc incarne le modèle de "perpétuelle conversation" et de "travail collaboratif" à l'échelle mondialisée qu'internet rend aujourd'hui accessible au plus grand nombre $»$.

La raison progresse par l'échange. C'était le pari des esprits éclairés à l'heure où Galilée se ralliait au système de Copernic. C'est aussi le modèle qui se déduit de la révolution numérique, quitte à s'y perdre dans le discours mêlé du prince, du savant, $\mathrm{du}$ poète, du marchand et... du fou. Le regard courroucé de Richelieu sur le PaloAlto aixois de Peiresc prouve au moins la vertu libérale de la méthode. 